Check for updates

Cite this: RSC Adv., 2019, 9, 19039

\title{
Stretchable self-healing hydrogels capable of heavy metal ion scavenging $\dagger$
}

\begin{abstract}
Dandan Song, Beibei Kang, Zengdian Zhao and Shasha Song (DD) *
Self-healing hydrogels were prepared by simply mixing phytic acid (PA) and chitosan (CS) in water. Determined by scanning electron microscopy (SEM), the hydrogels were found to be a threedimensional (3D) porous network structure. The formation of the network structure was considered to be mainly driven by electrostatic interactions and hydrogen bonding, cooperating with the subtle balance of multiple noncovalent interactions. The rheological data indicated that the hydrogels presented excellent mechanical properties with an elastic modulus of $20000 \mathrm{~Pa}$ and a yield stress exceeding $7000 \mathrm{~Pa}$. The dynamic dissociation and recombination of hydrogen bonding and electrostatic interaction in fractured regions of the gels initiated the self-healable property of PA/CS hydrogels. Since PA had high coordination ability to metal ions, PA/CS hydrogels were shown to exhibit excellent capability for capturing heavy metal ions, for example, $\mathrm{Pb}^{2+}$ and $\mathrm{Cd}^{2+}$. The PA/CS hydrogels provided a simple, green, and high efficiency strategic approach to scavenging heavy-metal ions from industrial sewage.
\end{abstract}

Received 8th May 2019

Accepted 29th May 2019

DOI: 10.1039/c9ra03443a

rsc.li/rsc-advances

molecular conformation is six-carbon ring with asymmetry,

\section{Introduction}

Hydrogels are soft and moist materials consisting of a threedimensional (3D) network cross-linked by covalent or noncovalent bonds, which have pervaded our daily life in diverse forms such as hair sprays, shampoo, toothpaste, contact lenses, paper diapers, and cosmetics. ${ }^{1}$ However, hydrogels always have poor mechanical properties, e.g. mechanical strength, viscoelasticity and fracture toughness, which prohibit their range of applications. Self-healing polymer hydrogels present dramatic changes in swelling behavior, mechanical strength, elastic modulus, permeability, and adhesion in response to external stimuli including ionic strength, $\mathrm{pH}$, temperature and magnetic field of the surrounding solution. ${ }^{2}$ Therefore, the self-healing polymer hydrogels have great potential in many fields e.g., tissue engineering, ${ }^{3,4}$ biomedical science, ${ }^{5-7}$ water remediation, ${ }^{\mathbf{8}, 9}$ supercapacitors, ${ }^{\mathbf{1 0 , 1 1}}$ shape memory devices, ${ }^{\mathbf{1 2}}$ and smart materials, ${ }^{2}$ and have drawn much attention in recent years. The properties of hydrogels can guide their application. Therefore, a deep knowledge of the properties of hydrogels (the structureproperty relationships) was highly desired in order to select a suitable material (shape and size) to be used in a given application. $^{2}$

Phytic acid (PA), a natural phosphorylated derivative, had significant physiological functions in plant life cycles. ${ }^{13}$ Its

School of Chemistry and Chemical Engineering, Shandong University of Technology, Zibo 255000, P. R. China. E-mail: songshasha@sdut.edu.cn

$\dagger$ Electronic supplementary information (ESI) available. See DOI: 10.1039/c9ra03443a which contains six phosphate esters and twelve hydroxyl groups, resulting in the higher coordination capacity under acidic and neutral conditions. Due to the unique chelation ${ }^{\mathbf{1 4}}$ and natural antioxidation property, ${ }^{15} \mathrm{PA}$ and its derivatives exhibit novel and abundant self-assembly behaviors, which have been gradually discovered in recent decades. ${ }^{16-19}$ In addition, PA has outstanding biocompatibility and biodegradability, which should be an excellent gelator to prepare functional gels for biological directed applications and environmental considerations. Regrettably, the gel formed by PA has been barely reported. Recently, contaminated water with heavy metal ions has been a growing problem due to the prohibition access to potable water. $^{\mathbf{2 0 2}}$ The vast majority heavy metal ions are produced in industrial processes, for example, electroplating, metal finishing, and mining. ${ }^{22}$ Different from organic wastewater, heavy metal ions are resistant and can be accumulated in environment and in body to evoke various diseases, for example, trace level of mercury can cause kidney, nervous system, and brain damage, ${ }^{21}$ intake of copper and nickel can destroy the liver, heart and kidneys and give rise to mental retardation. ${ }^{23}$ Therefore, an effective, cost-efficient, green and convenient approach is urgently required to purify wastewater and obtain freshwater resources. Due to the porous structure and large surface area, polymer hydrogels should be a promising adsorbent in scavenging of contaminants form water. ${ }^{24,25}$ However, the restriction of hydrogels utilized in the heavy metal ions removal is their insufficient mechanical integrity.

Herein, we report a self-healing polymer hydrogels with higher mechanical strength produced by mixing PA and 
chitosan (CS) in water. In the gels, both PA and CS are harmless environmentally materials with large number of active sites, which initiates the polymer gels efficient for removal of metal ions from industrial sewage. The polymer hydrogels was composed of 3D porous network structure driven by the subtle balance of multiple noncovalent interactions. In addition, the hydrogels exhibited excellent mechanical properties, which can be regulated by changing the proportion of the two compounds and the total concentration. The dynamic dissociation and recombination of hydrogen bonding and electrostatic interaction endow PA/CS hydrogels rapid self-healable behavior. The PA/CS hydrogels should be a promising adsorbent for scavenging of heavy metal ions from industrial sewage.

\section{Experimental section}

\subsection{Chemicals and materials}

Phytic acid (PA, $45 \mathrm{wt} \%$ ) was purchased from J\&K Chemical Company Ltd. (Beijing, China). Chitosan (CS) was purchased from Sigma-Aldrich (USA). Calcium chloride $\left(\mathrm{CaCl}_{2}\right)$, magnesium sulfate heptahydrate $\left(\mathrm{MgSO}_{4} \cdot 7 \mathrm{H}_{2} \mathrm{O}\right)$, lead(II) acetate trihydrate $\left(\mathrm{Pb}\left(\mathrm{CH}_{3} \mathrm{COO}\right)_{2} \cdot 3 \mathrm{H}_{2} \mathrm{O}\right)$, barium chloride dihydrate $\left(\mathrm{BaCl}_{2} \cdot 2 \mathrm{H}_{2} \mathrm{O}\right)$, copper(II) chloride dihydrate $\left(\mathrm{CuCl}_{2} \cdot 2 \mathrm{H}_{2} \mathrm{O}\right)$, ethylenediaminetetraacetic acid (EDTA), sodium hydroxide $(\mathrm{NaOH})$ and cadmium acetate dehydrate $\left(\mathrm{Cd}\left(\mathrm{CH}_{3} \mathrm{COO}\right)_{2} \cdot 2 \mathrm{H}_{2} \mathrm{O}\right)$ and xylenol orange (XO) were all purchased from Sinopharm Chemical Reagent Co. Ltd. (Shanghai, China) and were of p.a. quality. Ultrapure water used in the experiment was prepared by a UPH-IV ultrapure water purifier (Chengdu Ultrapure Technology Co. Ltd., China) with a resistivity of $18.25 \mathrm{M} \Omega \mathrm{cm}$.

\subsection{Sample preparation}

Different amounts of CS were accurately weighed and added into the test bottle, and then different concentrations of PA solution were added until the final volume of each sample reached $8 \mathrm{~mL}$. The sample was stirred mildly at $40{ }^{\circ} \mathrm{C}$ for $2 \mathrm{~h}$ until the CS dissolved completely. Then the samples were equilibrated at room temperature for more than four week until they were unchanged over an extended period of time.

\subsection{Field-emission scanning electron microscope observations}

For FE-SEM observation, a small volume of gel sample was coated on silicon wafer to form a film. The wafers were freezedried in a vacuum extractor at $-60{ }^{\circ} \mathrm{C}$ for several days. Then the silica wafers were observed at FEI Sirion 200 at $10 \mathrm{kv}$.

\subsection{Rheological measurements}

Rheological measurements were performed on a HAAKE RS6000 rheometer with a cone-plate system ( $\mathrm{Ti}$, diameter, 35 $\mathrm{mm}$; cone angle, $1^{\circ}$ ). The viscoelasticity of the sample was determined by the oscillatory measurements with an amplitude sweep in the frequency range of 0.01 to $10 \mathrm{~Hz}$. Prior to frequency sweep scanning frequency was fixed at $1.0 \mathrm{~Hz}$ in order to ensure the selected stress was in the linear viscoelastic region. The sample was measured at $25.0 \pm 0.1{ }^{\circ} \mathrm{C}$ with the help of a cyclic water bath.

\subsection{Fourier transform infrared spectra measurements}

The FT-IR spectra were carried out on a VERTEX-70/70v FT-IR spectrometer (Bruker Optics, Germany). FT-IR spectra were performed in the range of 4000-400 $\mathrm{cm}^{-1}$. The sample was mixed with $\mathrm{KBr}$ and pressed into a plate for the measurements.

\subsection{Metal ions absorption}

The solution of $\mathrm{Pb}^{2+}$ and $\mathrm{Cd}^{2+}\left(30 \mathrm{mg} \mathrm{mL}^{-1}\right)$ were prepared by dissolving solid $\mathrm{Pb}\left(\mathrm{CH}_{3} \mathrm{COO}\right)_{2} \cdot 3 \mathrm{H}_{2} \mathrm{O}$ and $\mathrm{Cd}\left(\mathrm{CH}_{3} \mathrm{COO}\right)_{2} \cdot 2 \mathrm{H}_{2} \mathrm{O}$ in water, respectively. PA/CS hydrogels were submerged into the solutions under stirring and then left undisturbed. The concentration variation of the heavy-metal ions after adsorption was quantified by UV-vis spectroscopy on an Agilent's Cary-60 spectrophotometer. The scan rate for each measurement was $600 \mathrm{~nm} \min ^{-1}$. Before the determination, xylenol orange was added to form the ligand complexes.

\section{Results and discussion}

\subsection{Gelation behavior}

Chitosan (CS) derived from deacetylated chitin was a natural amino homogeneous linear polysaccharide composed of glucosamine and $N$-acetyl-D-glucosamine units linked by the $\beta$ (1-4) glycosidic bond..$^{26,27} \mathrm{CS}$, an environmental friendly material, contained many active hydroxyl groups and free amino groups, which should be an excellent gelator to prepare functional biocompatible polymer hydrogels. ${ }^{28}$ Therefore, we investigated the gelation behavior of CS with phytic acid (PA). Fig. 1 gave the gel phase region in PA/CS system, which was confirmed by inverted tube observations. In this paper, we focus on the properties of the gel phase, including the gelation capability, microstructure and rheological properties. The gelation capability was appraised by critical gelation concentration (CGC), which can be calculated by the minimum amount of gelators to gelate $1 \mathrm{~mL}$ solvent or the minimum mass fraction of the gelators for the gel formation. ${ }^{29}$ Hydrogels can form at $c_{\mathrm{PA}}=$ $0.3 \mathrm{~mol} \mathrm{~L}^{-1}$ with $3 \mathrm{wt} \%$ of CS. For further detailed study, two series of samples were selected. One was at a fixed CS mass fraction of $6 \mathrm{wt} \%$ with various concentration of PA, while another was at a fixed PA concentration of $1 \mathrm{~mol} \mathrm{~L}^{-1}$ with different amount of CS. As shown in Fig. 1b, one can find that with the increase of CS (PA), the gel became opaque, which should be attributed to the microstructure transition.

\subsection{Microstructure of PA/CS gel}

FE-SEM was preformed to observe the morphology of PA/CS polymer hydrogels and to make an assessment of the influence of different composition, and shown in Fig. 2. The typical SEM images of the lyophilized PA/CS hydrogels presented heterogeneous porous microstructures, demonstrating the typical characteristic structures of polymer-based hydrogel. ${ }^{30}$ Generally, the presence of porous structure could promote the intrusion of water into polymeric network. ${ }^{5}$ Moreover, the 

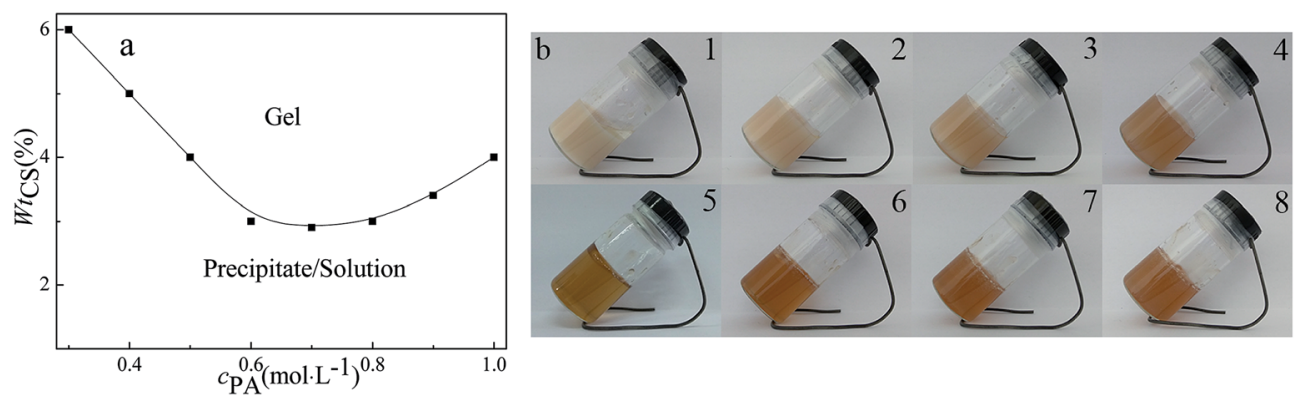

Fig. 1 (a) Phase diagram of PA/CS system at $25 \pm 0.1^{\circ} \mathrm{C}$. (b) Sample images at $w_{C S}=6$ wt $\%$ with different concentrations of PA (mol $L^{-1}$ ) of (1) 0.3 , (2) 0.5 , (3) 0.6 , (4) 0.8 , (5) 1; at $C_{\mathrm{PA}}=1 \mathrm{~mol} \mathrm{~L}^{-1}$ with different mass fraction of CS (wt\%) of (6) 5, (7) 7, (8) 8.

internal morphology and pore sizes interpreted the influence of the content of PA (CS) on the cross-linking density. As shown in Fig. 2a and b, the porous structure of PA/CS hydrogels was disorderly arranged with random pores size in the range of 20 to $100 \mu \mathrm{m}$. At a fixed CS concentration of $6 \mathrm{wt} \%$, when PA reached $0.6 \mathrm{~mol} \mathrm{~L}^{-1}$, hydrogels presented a honeycomb-like structure consisting of orderly arranged pores with an average size of approximately $20 \mu \mathrm{m}$ and the wall of the pores appeared to be thinner (Fig. 2c). When PA concentration was above $0.8 \mathrm{~mol} \mathrm{~L}^{-1}$, the porous structure became disorderly, and the diameter of the pored declined to approximately $15 \mu \mathrm{m}$ (Fig. 2d). With the increase of PA, the microporous morphology became much more irregular in both size and distribution and the wall of the pores appeared to be thinner.

The morphology variation of another series samples with same amounts of PA but different content of CS was shown in Fig. 2e-h. With detailed observation of the SEM images, one can find that with increasing the amount of CS, the porous microstructures were distributed much more orderly, and the diameter of the pored declined to approximately $15 \mu \mathrm{m}$. The reason might be that lower CS or PA content in the polymer hydrogels exhibited higher water content, which stimulated the generation of a larger ice crystal when immersing the hydrogels into liquid nitrogen. ${ }^{5}$ These larger ice crystals were lyophilization and eventually, bigger pores were produced. Taken together, the morphology of PA/CS polymer hydrogels could be regulated by adjusting the content of PA or CS in the gels. Changes in morphology of the hydrogels may involve differences in the rheological property, which would be discussed as following.

\subsection{Rheological properties}

The rheological data (mechanical strength and viscoelasticity) can not reflect the cross-linking density of the microstructure, but provide guidelines for gel applications. The mechanical properties of $\mathrm{PA} / \mathrm{CS}$ hydrogels were studied by dynamic rheology, which depicts elastic modulus $\left(G^{\prime}\right)$ as a function of shear stress $(\tau)$ at a fixed frequency of $1 \mathrm{~Hz}$. Fig. 3 present the rheological properties for PA/CS gels at different amounts of PA or CS. The yield stress $\left(\tau^{*}\right)$ is the stress in oscillatory mode where the $G^{\prime}$ decrease suddenly, indicating the hydrogels networks have been destroyed at this stress. At a fixed CS concentration of $6 \mathrm{wt} \%$, with the increase in PA concentration from 0.3 to $1.0 \mathrm{~mol} \mathrm{~L}^{-1}$, the $\tau^{*}$ increase gradually from 2000 to $4000 \mathrm{~Pa}$ and the $G^{\prime}$ increase from 10000 to $20000 \mathrm{~Pa}$ first, which was higher than other reported polymer hydrogels, ${ }^{20,31,32}$ exhibiting excellent mechanical strength. With further increase in PA concentration, the $\tau^{*}$ and $G^{\prime}$ value dropped. The rheological property was in accord with the changes in morphology of the polymer gels. At a lower PA concentration, the microstructure was composed of disorderly pores with lower crosslinking density, leading to a weaker mechanical strength.
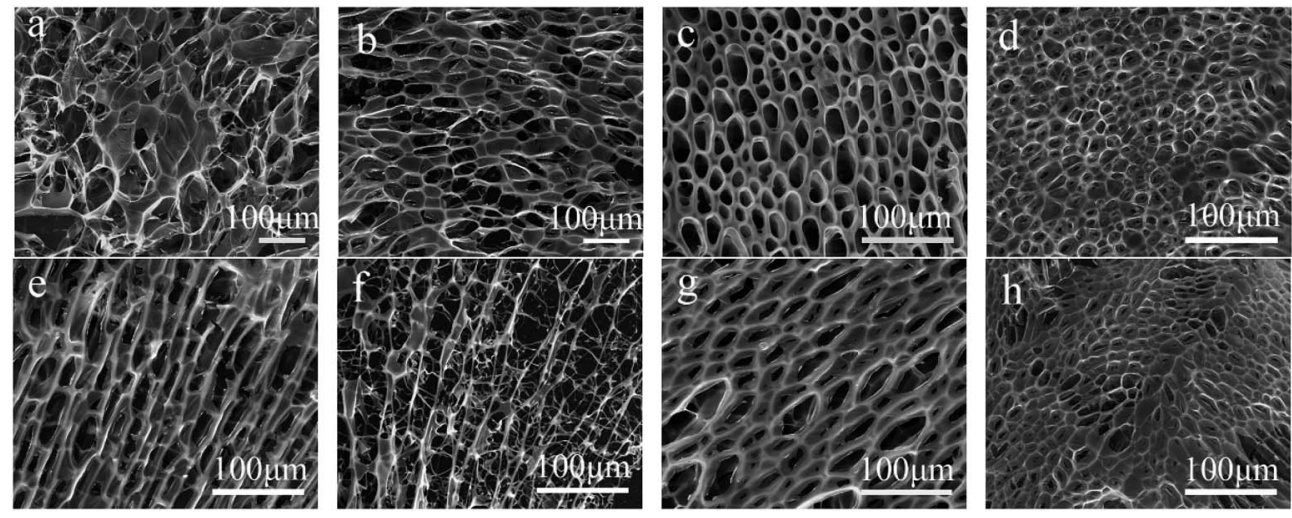

Fig. 2 FE-SEM images of the samples formed by 6 wt\% CS with changeable $C_{P A}\left(\mathrm{~mol} \mathrm{~L}^{-1}\right)$ : (a) 0.3 , (b) 0.5 , (c) 0.6 , (d) 0.8 , (e) 1 ; and by 1 mol L ${ }^{-1}$ PA with changeable $w_{C S}(w t \%)$ : (f) 5, (g) 7, (h) 8 . 

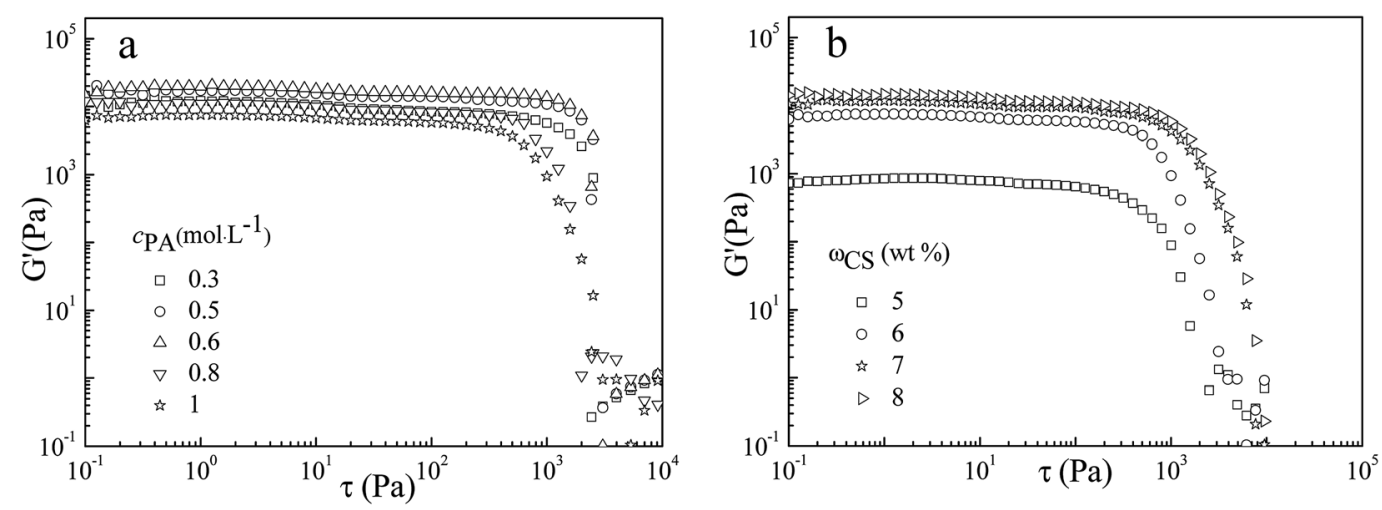

Fig. 3 Elastic modulus $\left(G^{\prime}\right)$ as a function of shear stress $(\tau)$ of gels formed (a) at $w_{C S}=6$ wt $\%$ with different $c_{P A}$, (b) at $c_{P A}=1$ mol $L^{-1}$ with different $w_{C S}$.

When $c_{\mathrm{PA}}=0.6 \mathrm{~mol} \mathrm{~L}^{-1}$, the hydrogels showed the homogeneous porous microstructures with the highest yield stress and elastic modulus, suggesting the higher crosslinking density. At $c_{\mathrm{PA}}>0.6 \mathrm{~mol} \mathrm{~L}^{-1}$, the internal morphology and pore sizes of the gels became irregularly, corresponding to the decrease in mechanical strength. For another series sample, at a fixed PA concentration of $1.0 \mathrm{~mol} \mathrm{~L}^{-1}$, with the addition of $\mathrm{CS}$, the porous structure became orderly, leading to the increase of $\tau^{*}$ and $G^{\prime}$, as shown in Fig. 3b.

Viscoelasticity can be characterized by determined the elastic modulus $\left(G^{\prime}\right.$, evaluating the degree of resistance against mechanical disturbance) and the viscous modulus ( $G^{\prime \prime}$, estimating the tendency of materials to flow under stress) as a function of frequency with a fixed shear stress of $10 \mathrm{~Pa}$. As shown in Fig. 4, the $G^{\prime}$ was higher than $G^{\prime \prime}$ in the test frequency range (0.1-10 Hz) for each sample, and the values of $G^{\prime}$ and $G^{\prime \prime}$ slightly increased with frequency, indicating the dominant elastic character, which was typically observed for polymer gels. ${ }^{9}$ Owing to the microstructure transition, the value of $G^{\prime}$ and $G^{\prime \prime}$ increased with the increasing in PA or CS concentration. Based on the microstructure and rheological results, we can conclude that the morphological, rheological and mechanical properties of obtained hydrogels could be readily controlled by regulating the amount of gelators.

\subsection{Formation mechanism of PA/CS hydrogels}

The formation of cross-linking network structures was very essential for the gelation. ${ }^{33}$ FT-IR was carried out to interpret the formation mechanism of network structure in $\mathrm{PA} / \mathrm{CS}$ polymer gels. PA was a natural organic hexaphosphate, and which $-\mathrm{P}=\mathrm{O}$ group had an obvious stretching vibration peak at $1126 \mathrm{~cm}^{-1} \cdot{ }^{16,34}$ When PA/CS gels was formed, the stretching peak of $-\mathrm{P}=\mathrm{O}$ group at $1126 \mathrm{~cm}^{-1}$ disappeared and split into two peaks at $1134 \mathrm{~cm}^{-1}$ (asymmetric stretching frequency) and $1166 \mathrm{~cm}^{-1}$ (symmetric stretching frequency), which can be ascribed to the phosphoric acid of PA converted into phosphate with the $-\mathrm{NH}_{3}{ }^{+}$of $\mathrm{CS}$ as the counterions. Moreover, the stretching frequency of $-\mathrm{NH}_{2}$ group of $\mathrm{CS}$ at $\tilde{v}=3434 \mathrm{~cm}^{-1}$ became sharping, which further confirmed the $-\mathrm{NH}_{2}$ of $\mathrm{CS}$ had been protonated by the phosphoric acid of PA. The stretching mode of $-\mathrm{P}-\mathrm{O}$ at $1020 \mathrm{~cm}^{-1}$ split into two peaks: antisymmetrical frequency $\left(1060 \mathrm{~cm}^{-1}\right)$ and symmetrical frequency $\left(1010 \mathrm{~cm}^{-1}\right)$ (Fig. 5a, curves 1 and 2). This could be attributed to the formation of hydrogen bonding between PA and CS, which promote the stretching vibrational mode of $-\mathrm{P}-\mathrm{O}$ group, causing the split and bathochromic shift of the absorption peak. In PA/CS hydrogels, between PA and CS contained similar intermolecular interaction, no matter the change in PA or CS concentration (Fig. S1†).

According to the microstructure and FT-IR results, the formation mechanism of the cross-linking porous structure in PA/CS hydrogels was proposed and shown in Fig. 5b. When CS was added to PA solution, the $-\mathrm{NH}_{2}$ of $\mathrm{CS}$ was protonated by the phosphoric acid of PA to form $-\mathrm{NH}_{3}{ }^{+} \ldots \mathrm{PO}_{4} \mathrm{H}^{-}$ion pairs. In addition to electrostatic interactions between $-\mathrm{NH}_{3}{ }^{+} \cdots \mathrm{PO}_{4} \mathrm{H}^{-}$ ion pairs, hydrogen bonding also existed between the $-\mathrm{O}-\mathrm{H}$ of CS and -P-O of PA. With the assistance of a delicate balance of hydrophobic interaction and van der waal forces, porous network structure was formed, which immobilized water molecules, leading to the formation of polymer hydrogels with excellent mechanical strength.

\subsection{Multiresponsiveness of PA/CS hydrogels}

The cross-linking pores in hydrogels was speculated to be mainly driven by the electrostatic interaction and hydrogen bonding between PA and CS, accompanied by the assistance of multiple noncovalent interaction including hydrophobic interaction and van der waal forces. External stimuli could shift the equilibrium of noncovalent interaction in the hydrogels, leading to the liquefaction of the hydrogels. Temperature, oscillating force, and heavy metal ions stimuli were performed to liquefy the hydrogels (Fig. 6a). When oscillating force was introduced into the hydrogels, the balance of noncovalent interaction would be break, resulting in the degradation of the hydrogels. Hydrogels would be regenerated in $2 \mathrm{~h}$ after removing the oscillating force. Hydrogen bonding was sensitive to temperature. Thus, when heated, the hydrogels was liquefied to solution due to the destroy of hydrogen bonding between PA and CS, and the solution can turn back into gel in several hours 

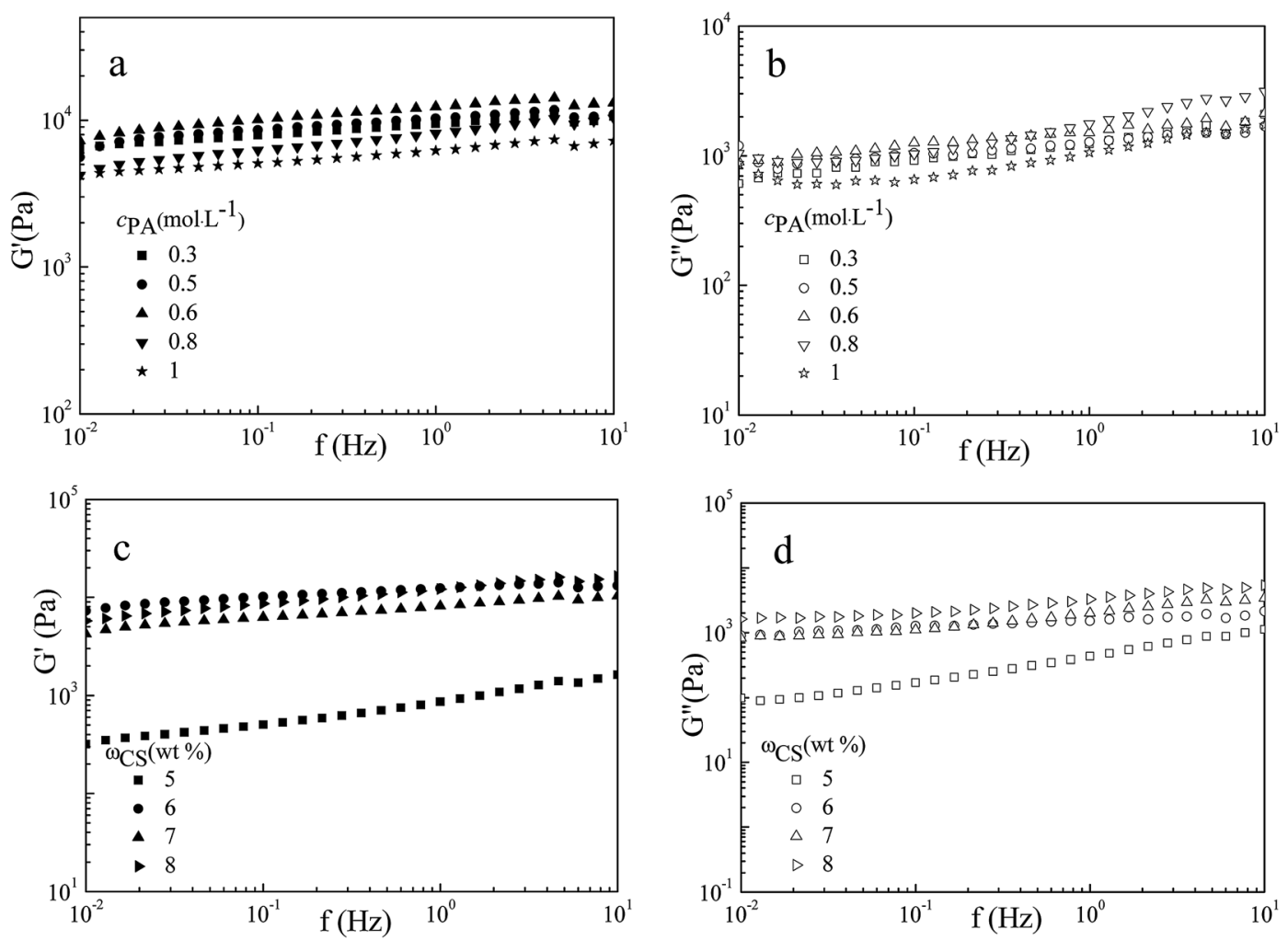

Fig. 4 Elastic modulus $\left(G^{\prime}\right)$ as a function of frequency of gels formed (a) at $w_{C S}=6$ wt $\%$ with different $C_{P A}$, (c) at $C_{P A}=1$ mol $L^{-1}$ with different $w_{C S}$; viscous modulus $\left(G^{\prime \prime}\right)$ as a function of frequency of gels formed $(b)$ at $w_{C S}=6$ wt $\%$ with different $C_{P A}$, (d) at $C_{P A}=1 \mathrm{~mol} \mathrm{~L} L^{-1}$ with different $w_{C S}$.

once cooled to room temperature since to the recovery of hydrogen bonding.

PA, a cyclic acidic molecule saturated with six dihydrogen phosphate, exerted a strong binding affinity to metallic ions, and the capability of chelate bivalent cations would be increased through inducing phosphate variants in a deprotonating environment. ${ }^{34}$ Metallic ions can generate more stable coordination compound with $\mathrm{PA}$ than the $-\mathrm{NH}_{3}{ }^{+} \cdots \mathrm{PO}_{4} \mathrm{H}^{-}$ion pairs between PA and CS. As a result, the metal ions $\left(\mathrm{Ca}^{2+}, \mathrm{Cu}^{2+}\right.$, $\mathrm{Mg}^{2+}, \mathrm{Pb}^{2+}$ and $\mathrm{Ba}^{2+}$ ) added to the hydrogels would combine with PA replace the intermolecular interaction between PA and CS, which disrupted the hydrogel structure, leading to the degradation of the hydrogel in about $2 \mathrm{~h}$ (Fig. $6 \mathrm{a}$ and $\mathrm{S} 2 \dagger$ ). When a complexing agent, disodium salt of ethylenediaminetetraacetic acid (EDTA-2Na), was added to the solution, hydrogels were regenerated in $30 \mathrm{~min}$. The reason might be that the complexing constant between metal ion and EDTA, $K_{(\mathrm{MY})}$, was much larger than between metal ions and PA, $K_{(\mathrm{MPA})} \cdot^{\mathbf{1 6 , 3 4 , 3 5}}$ Therefore, the PA was released and the equilibrium of noncovalent interaction between PA and CS reformed, resulting in the recovery of hydrogels. It was worth mentioning that the regenerated hydrogel still exhibited higher mechanical strength and elastic character dominant behavior, as shown in Fig. 6b and $c$.
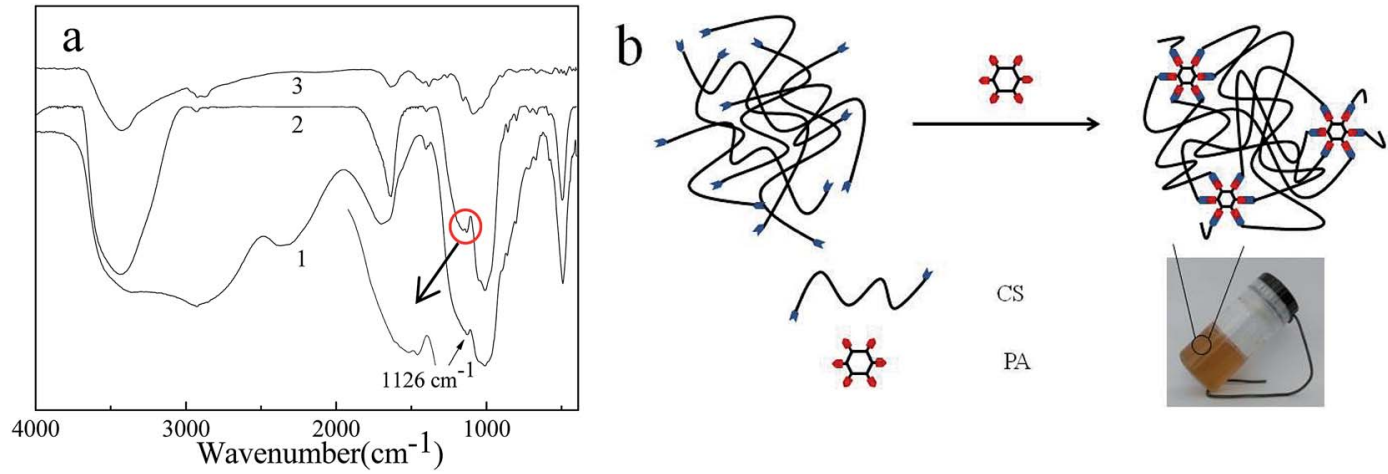

Fig. 5 (a) FT-IR spectra of curve (1) PA, curve (2) 0.6 mol L ${ }^{-1} P A / 6$ wt\% CS, curve (3) CS. (b) Schematic representation of the porous structure formed in PA/CS hydrogels. 
a

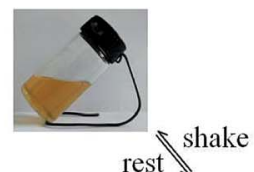

rest 1
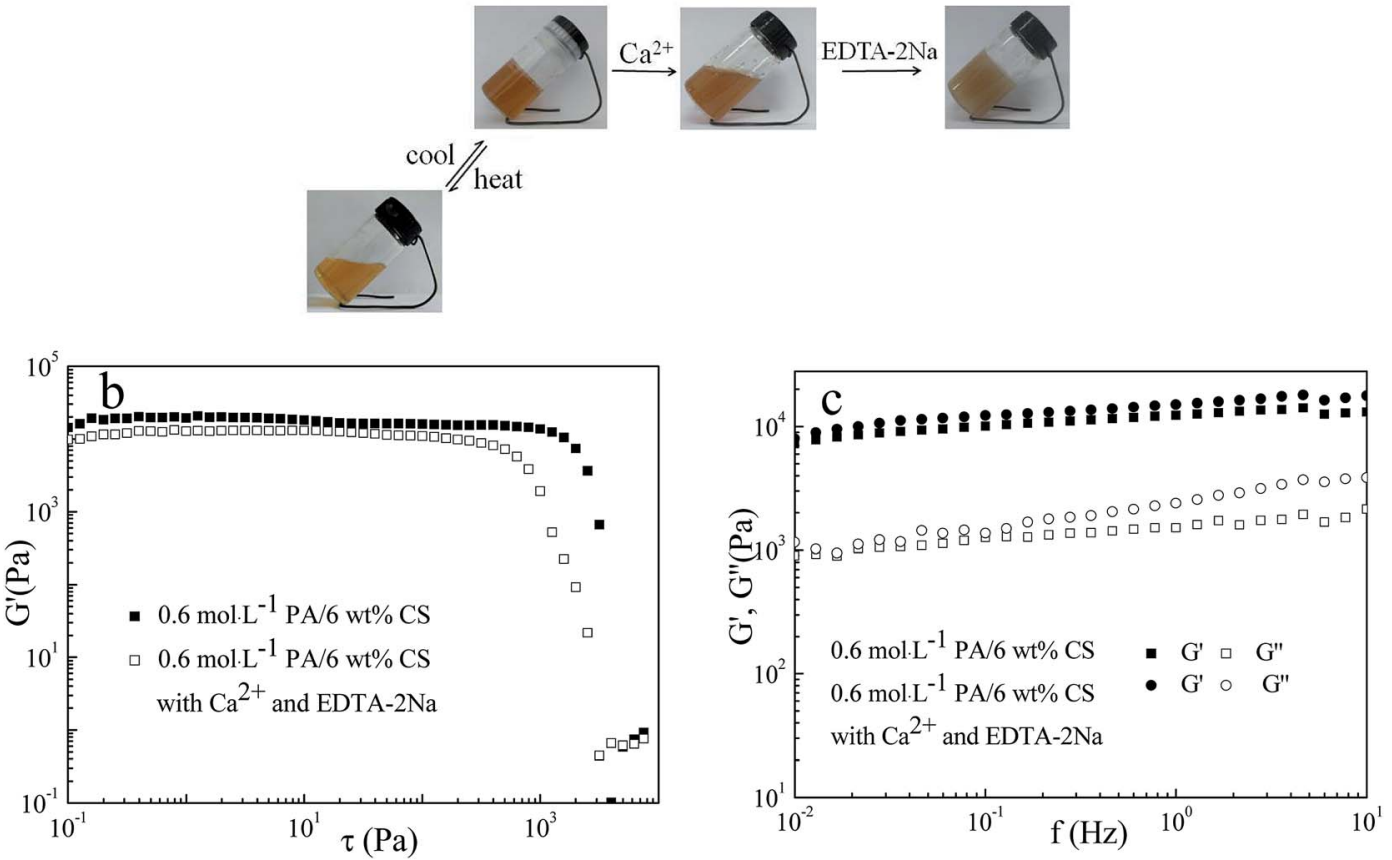

Fig. 6 (a) Hydrogel's multiresponses to different stimuli, (b) elastic modulus $\left(G^{\prime}\right)$ as a function of shear stress of PA/CS hydrogels, (c) elastic modulus $\left(G^{\prime}\right)$ and viscous modulus $\left(G^{\prime \prime}\right)$ as a function of frequency of PA/CS hydrogels.

\subsection{Self-healing property}

The scope of hydrogels application was always severely limited by their mechanical behavior. ${ }^{36,37}$ Most hydrogels did not present good stretchability, i.e., hydrogel ruptured when stretched to about 1-2 times to its original length. However, in our experiment, we found that $\mathrm{PA} / \mathrm{CS}$ polymer hydrogels exhibited well tensile property (see Fig. 7a and Movie S1†). The hydrogels remained its initial flat configuration after elongated about 2 times to its premier length.

Intelligent gels with self-healing or self-repairing properties can restore their functionalities and structures after damage, which extend the service life and improve the used safety of the materials, being the cutting-edge topics in gel materials. ${ }^{38}$
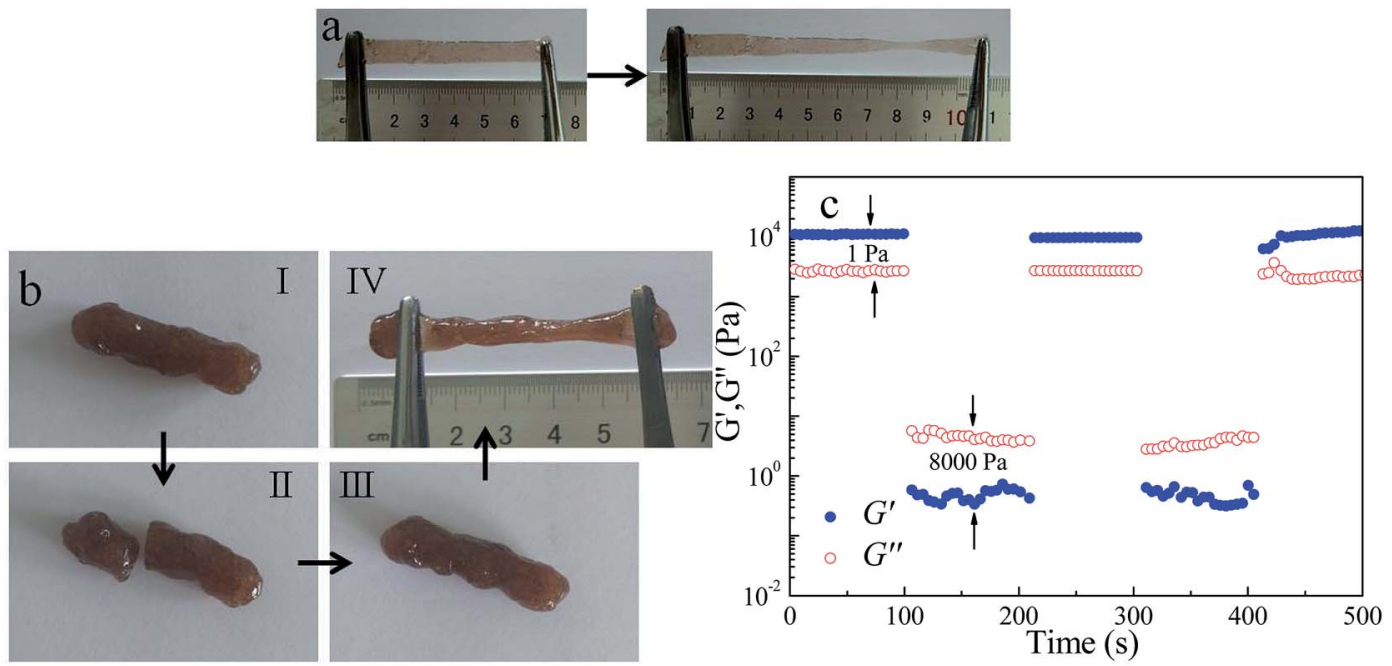

Fig. 7 (a) Visual photos used to show the excellent ductility. (b) Optical evidence of self-healing: (I) initial sample (0.6 mol L ${ }^{-1}$ PA/6 wt\% CS), (II) cut sample, (III) cut sample rejoined for healing, and (IV) self-healed sample with a stretchable stress. (c) $G^{\prime}$ and $G^{\prime \prime}$ value of the 0.6 mol $L^{-1}$ PA/ 6 wt\% CS hydrogel as alternate step stress switched from small stress ( $1 \mathrm{~Pa})$ to large stress (8000 Pa) with a fixed $100 \mathrm{~s}$ interval. 

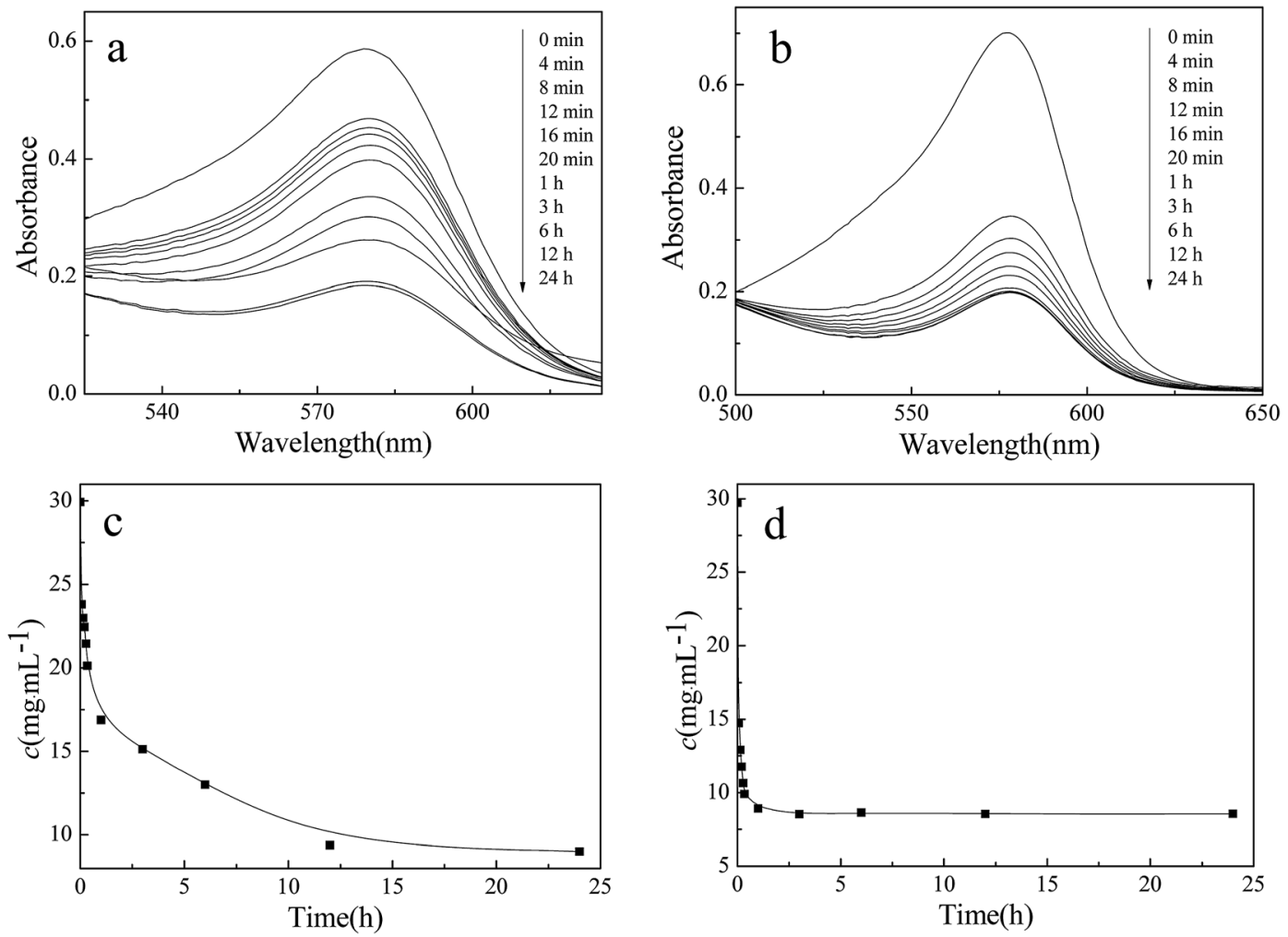

Fig. 8 UV-vis spectra of solutions of (a) $\mathrm{Pb}^{2+}$ and (b) $\mathrm{Cd}^{2+}$ with time after the addition of the PA/CS hydrogels $(4.0 \mathrm{~g})$. The concentration variation of (c) $\mathrm{Pb}^{2+}$ and (d) $\mathrm{Cd}^{2+}$ as a function of time.

Physical self-healing gels restored network through dynamic formation of attractive noncovalent interactions between molecule or polymer chains, including hydrophobic interactions, ${ }^{39,40}$ host-guest interactions, ${ }^{31}$ hydrogen bonding, ${ }^{41}$ crystallization, ${ }^{42}$ and multiple intermolecular interactions. ${ }^{43}$ The electrostatic interactions and hydrogen bonding between PA/CS acted a crucial role in the gel formation, endowed the hydrogels self-healable character. Macroscopic self-healing test were carried out to visually evaluate the self-healing ability of PA/CS hydrogels, as shown in Fig. 7b. After the gel was cut and rejoined, it repaired itself within less than $5 \mathrm{~min}$ without any external intervention. Interestingly, the healed hydrogel can be stretched, suggesting high self-healing efficiency. The dynamic hydrogen bonding and electrostatic interaction between PA and CS lead to a quick recovery, healing the ruptured pieces rapidly at the interfaces.

Oscillatory rheological characterization was conducted to quantification the self-healing behavior of PA/CS hydrogels. As shown in Fig. 7c, the step shear stress measurements under the alternating small $(1 \mathrm{~Pa})$ and larger (8000 Pa) were carried out. Under a small stress, $G^{\prime}$ was larger than $G^{\prime \prime}$, demonstrating a denser cross-linking network in the hydrogels. However, a sudden increased stress from 1 to $8000 \mathrm{~Pa}$ caused gel failure, accompanied by a decrease in $G^{\prime}$ and $G^{\prime \prime}$ and the values are inverted, suggesting a solution state formed. When stress was brought to $1 \mathrm{~Pa}, G^{\prime}$ and $G^{\prime \prime}$ could fast recovered to their original values within a few seconds. The repeatability of this transition process was confirmed by the second cycles. All the results presented that PA/CS hydrogels possessed a rapid self-healing capability due to the dissociation and recombination of dynamic physical interactions (hydrogen bonding and electrostatic interaction).

\subsection{Heavy metal ions adsorption}

Generally, the presence of porous structure could facilitate the penetration of water into polymeric network, consequently profiting for the adsorbency of aqueous. ${ }^{20}$ In addition, PA can

Table 1 Scavenging capability and concentration of heavy metal ion in the mixed solution before and after the adsorbent PA/CS gel treatment and the partition coefficients

\begin{tabular}{llll}
\hline $\begin{array}{l}\text { Heavy metal } \\
\text { ion }\end{array}$ & $C_{\mathrm{i}}\left(\mathrm{mg} \mathrm{mL}^{-1}\right)$ & $C_{\mathrm{e}}\left(\mathrm{mg} \mathrm{mL}^{-1}\right)$ & \multicolumn{2}{l}{$\begin{array}{l}\text { Scavenging capacity } \\
\left(\mathrm{mg} \mathrm{g}^{-1}\right)\end{array}$} \\
\hline $\mathrm{Pb}^{2+}$ & 29.9298 & 9.0061 & 1750.66 \\
$\mathrm{Cd}^{2+}$ & 29.7362 & 8.5524 & 1772.4
\end{tabular}




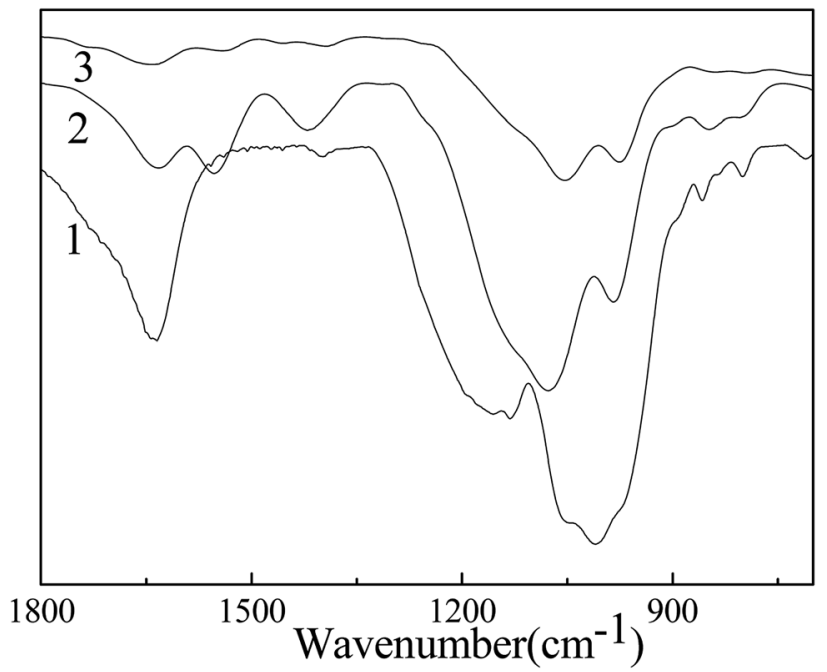

Fig. 9 FT-IR spectra of PA/CS hydrogels (curves (1)), the hydrogel samples after adsorption of $\mathrm{Pb}^{2+}$ (curves (2)) and $\mathrm{Cd}^{2+}$ (curves (3)).

interact with metallic bivalent ions to form stable complexes, which effectively inhibit the oxidative DNA damage and the generation of highly reactive species from $\mathrm{H}_{2} \mathrm{O}_{2}$, resulting in chemoprevention of cancer. ${ }^{44-46}$ Therefore, PA/CS hydrogels were expected to function as efficient metal ion scavengers. To examine metal-ion uptake by the PA/CS hydrogels, $4.0 \mathrm{~g}$ PA/CS hydrogels was immersed into aqueous solutions of $\mathrm{Pb}^{2+}$ and $\mathrm{Cd}^{2+}(250 \mathrm{~mL})$ solutions without being disturbed. Fig. 8 presented the concentration variation of $\mathrm{Pb}^{2+}$ and $\mathrm{Cd}^{2+}$ as a function of adsorption time. It was clearly seen that the concentration of $\mathrm{Pb}^{2+}$ and $\mathrm{Cd}^{2+}$ rapidly decreased during the initial $12 \mathrm{~min}$, and then the concentration reached stable values after $12 \mathrm{~h}$.

In order to determine metal ion scavenging quantitatively, scavenging capacity of PA/CS hydrogels was calculated, which was determined as:

$$
\text { Scavenging capacity }=\frac{W_{\mathrm{i}}}{W_{\mathrm{g}}}
$$

where $W_{\mathrm{i}}$ is the weight of metal ions adsorbed by the hydrogels, and $W_{\mathrm{g}}$ is the mass of the hydrogels. ${ }^{20}$ As shown in Table 1 , the scavenging capacity of the $\mathrm{PA} / \mathrm{CS}$ hydrogels for $\mathrm{Pb}^{2+}$ and $\mathrm{Cd}^{2+}$ were $1750.66 \mathrm{mg} \mathrm{g}^{-1}$ and $1772.4 \mathrm{mg} \mathrm{g}^{-1}$, respectively, which was notably higher than the reported gels..$^{\mathbf{2 0 , 2 4 , 2 5}}$ The partition coefficient $(K)$ could express the adsorption capability of adsorbent to metal ions, which is defined as the ratio of the amount of metal ions adsorbed by the adsorbent in aqueous solutions:

$$
K=\frac{C_{\mathrm{i}}-C_{\mathrm{e}}}{C_{\mathrm{e}}} \times \frac{V(\mathrm{~mL})}{M(\mathrm{~g})}
$$

in which $C_{\mathrm{i}}$ and $C_{\mathrm{e}}$ are the initial and equilibrium concentration of heavy metal ions, respectively; $V$ is the volume of the heavy metal ions solution; and $M$ is the mass of the adsorbent. ${ }^{22,47}$ Table 1 presents the partition coefficient $(K)$ of PA/CS hydrogels adsorbing the heavy metal ions. From the partition coefficient $(K)$ and the scavenging capacity of PA/CS hydrogels, we can conclude that PA/CS hydrogels exhibited superior adsorption capability to metal ions, which can be used for scavenging of metal ions from their aqueous solutions.

To interpret the superior scavenging capability of PA/CS hydrogels for metal ions, FT-IR spectra of the hydrogels before and after the adsorption of metal ions were conducted and shown in Fig. 9. For $\mathrm{PA} / \mathrm{CS}$ hydrogels, $-\mathrm{P}=\mathrm{O}$ group has two stretching peaks: asymmetric stretching frequency $\left(1134 \mathrm{~cm}^{-1}\right)$ and symmetric stretching frequency $\left(1166 \mathrm{~cm}^{-1}\right)$. After the adsorption of metal ions, the stretching frequencies of $-\mathrm{P}=\mathrm{O}$ group disappeared. In addition, the antisymmetrical frequency of $-\mathrm{P}-\mathrm{O}$ at $1060 \mathrm{~cm}^{-1}$ shift to a higher wavenumber and the symmetrical frequency of $-\mathrm{P}-\mathrm{O}$ at $1010 \mathrm{~cm}^{-1}$ move to a lower wavenumber, which indicated the coordination interaction between PA and metal ions, and resulted in the predominant removal capacity for metal ions.

\section{Conclusions}

Self-healing polymer hydrogels with multiresponsiveness were successfully produced by mixing PA and CS in water. The main driving force in the gelation is the electrostatic interaction and hydrogen bonding between PA and CS. The dissociation and recombination of dynamic physical interaction, hydrogen bonding and electrostatic interaction, impart the hydrogels rapid self-healable characteristics, which was confirmed by the macroscopic self-healing test and oscillatory rheological measurement. The hydrogels possess interconnected porous structure, excellent ductility and good mechanical properties, which can be readily controlled by regulating the amount of gelators. Owing to the large number of active sites coordinating to metal ions and the porous structure of PA/CS hydrogels, the gels could efficiently and quickly adsorb heavy metal ions in an environmental friendly approach. We belive that the stretchable, rapid self-healing PA/CS hydrogels with superior combining capability for heavy metal ions can be a potential candidate for the scavenging of heavy metals from polluted water and the collection of metal ions form aqueous.

\section{Conflicts of interest}

There are no conflicts to declare.

\section{Acknowledgements}

This work is financially supported by the NSFC (21603123), the Opening Project of Oil \& Gas Field Applied Chemistry Key Laboratory of Sichuan Province (KF201405).

\section{References}

1 F. Wang and R. Weiss, Macromolecules, 2018, 51, 7556-7566. 2 D. Caccavo, S. Cascone, G. Lamberti and A. Barba, Chem. Soc. Rev., 2018, 47, 2357-2373.

3 Z. Zhang, X. Wang, Y. Wang and J. Hao, Biomacromolecules, 2018, 19, 980-988. 
4 D. Wang, Y. Sun, M. Cao, J. Wang and J. Hao, RSC Adv., 2015, 5, 95604-95612.

5 X. Qi, Y. Yuan, J. Zhang, J. Bulte and W. Dong, J. Agric. Food Chem., 2018, 66, 10479-10489.

6 X. Ding and Y. Wang, J. Mater. Chem. B, 2017, 5, 887-906.

7 D. Caccavo, S. Cascone, G. Lamberti, A. Barba and A. Larsson, Curr. Drug Delivery, 2017, 14, 179-189.

8 M. Zhang, R. Wang, Z. Shi, X. Huang, W. Zhao and C. Zhao, J. Hazard. Mater., 2017, 322, 499-507.

9 Y. Liu, D. Song, S. Song and Z. Zhao, ChemistrySelect, 2018, 3, 7310-7317.

10 X. Wu and A. Xu, J. Mater. Chem. A, 2014, 2, 4852-4864.

11 Q. Chen, H. Lu, F. Chen, L. Chen, N. Zhang and M. Ma, ACS Appl. Energy Mater., 2018, 1, 4261-4268.

12 D. Habault, H. Zhang and Y. Zhao, Chem. Soc. Rev., 2013, 42, 7244-7256.

13 J. Zhou and J. Erdman, Crit. Rev. Food Sci. Nutr., 1995, 35, 495-508.

14 R. Jariwalla, Drugs Exp. Clin. Res., 2001, 27, 17-26.

15 C. Kunyanga, J. Imungi, M. Okoth, H. Biesalski and V. Vadivel, Ecol. Food Nutr., 2011, 50, 452-471.

16 H. Kim, S. Im, J. Kim, W. Hong, K. Shin, H. Jeong and Y. Hong, ACS Sustainable Chem. Eng., 2017, 5, 6654-6664.

17 L. Caseli, M. Moraes, V. Zucolotto, M. Ferreira, T. Nobre, M. Zaniquelli, U. Fiho and O. Oliveira, Langmuir, 2006, 22, 8501-8508.

18 J. Su, X. Wu, C. Yang, J. Lee, J. Kim and Y. Guo, J. Phys. Chem. C, 2012, 116, 5019-5024.

19 H. Lei, N. Pan, X. Wang and H. Zou, J. Chem. Eng. Data, 2018, 63, 3989-3997.

20 A. Moien, K. Nancy, K. Amir, H. Andria, T. Moritz, S. Toyoko and K. Eugenia, ACS Nano, 2018, 12, 8160-8168.

21 M. Barakat, Arabian J. Chem., 2011, 4, 361-377.

22 S. Song, H. Wang, A. Song and J. Hao, Chem.-Asian J., 2014, 9, 245-252.

23 S. Needhidasan, M. Samuel and R. Chidambaram, J. Environ. Health Sci. Eng., 2014, 12(1-9), 36.

24 L. Wang and M. Wang, ACS Sustainable Chem. Eng., 2016, 4, 2830-2837.

25 B. Okesola and D. Smith, Chem. Soc. Rev., 2016, 45, 42264251.

26 J. Fu, F. Yang and Z. Guo, New J. Chem., 2018, 42, 1716217180.
27 T. Kiang, J. Wen, H. Lim and K. Leong, Biomaterials, 2004, 35, 5293-5301.

28 R. Jayakumar, D. Menon, K. Manzoor, S. Nair and H. Tamura, Carbohydr. Polym., 2010, 82, 227-232.

29 S. Song, Y. Liu, A. Song, Z. Zhao, H. Lu and J. Hao, J. Colloid Interface Sci., 2017, 506, 46-57.

30 R. Guo, Q. Su, J. Zhang, A. Dong, C. Lin and J. Zhang, Biomacromolecules, 2018, 18, 1356-1364.

31 Y. Jia, J. Jin, S. Liu, L. Ren, J. Luo and X. Zhu, Biomacromolecules, 2018, 19, 626-632.

32 M. Pandey, N. Mohamad and M. Amin, Mol. Pharmaceutics, 2014, 11, 3596-3608.

33 Y. Pan, B. Li, Z. Liu, Z. Yang, X. Yang, K. Shi, W. Li, C. Peng, W. Wang and X. Ji, ACS Appl. Mater. Interfaces, 2018, 10, 32747-32759.

34 L. Carli, E. Schnitzler, M. Ionashiro, B. Szpoganicz and N. Rosso, J. Braz. Chem. Soc., 2009, 20, 1515-1522.

35 K. Blomqvist and E. Still, Inorg. Chem., 1984, 23, 3730-3734. 36 P. Calvert, Adv. Mater., 2009, 21, 743-756.

37 J. Sun, X. Zhao, W. Illeperuma, O. Chaudhuri, K. Oh, D. Mooney, J. Vlassak and Z. Suo, Nature, 2012, 489, 133-136.

38 Z. Wei, J. Yang, J. Zhou, F. Xu, M. Zrinyi, P. Dussault, Y. Osada and Y. Chen, Chem. Soc. Rev., 2014, 43, 8114-8131.

39 G. Akay, A. Raeisi, D. Tuncaboylu, N. Orakdogen, S. Abdurrahmanoglu, W. Oppermann and O. Okay, Soft Matter, 2013, 9, 2254-2261.

40 D. Tuncaboylu, A. Argun, M. Sahin, M. Sari and O. Okay, Polymer, 2012, 53, 5513-5522.

41 A. Phadke, C. Zhang, B. Arman, C. Hsu, R. Mashelkar, A. Lele, M. Tauber, G. Arya and S. Varghese, Proc. Natl. Acad. Sci. U. S. A., 2012, 109, 4383-4388.

42 H. Zhang, H. Xia and Y. Zhao, ACS Macro Lett., 2012, 1, 12331236.

43 X. Yu, X. Cao, L. Chen, H. Lan, B. Liu and T. Yi, Soft Matter, 2012, 8, 3329-3334.

44 E. Graf, J. Am. Oil Chem. Soc., 1983, 60, 1861-1867.

45 P. Le Francois, J. Food Compos. Anal., 1988, 1, 139-145.

46 K. Midorikawa, M. Murata, S. Oikawa, Y. Hiraku and S. Kawanishi, Biochem. Biophys. Res. Commun., 2001, 288, 552-557.

47 D. Liu, J. Lei, L. Guo, X. Du and K. Zeng, Microporous Mesoporous Mater., 2019, 117, 67-74. 